\title{
Eficácia do mentol como anestésico para juvenis de tilápia-do-nilo (Oreochromis niloticus)
}

\author{
[Menthol efficiency as anesthetic for juveniles Nile tilapia Oreochromis niloticus] \\ L.N. Simões, L.C. Gomes* \\ Centro Universitário Vila Velha \\ Rua Comissário José Dantas de Melo, 21 - Boa Vista \\ 29102-770 - Vila Velha, ES
}

\begin{abstract}
RESUMO
Em três experimentos, testou-se a eficiência do mentol como anestésico para a tilápia-do-nilo (Oreochromis niloticus), por meio da avaliação de eventos comportamentais, da verificação da margem de segurança do anestésico e das respostas de estresse. No primeiro experimento, foram testadas seis diferentes concentrações de mentol para avaliar o tempo de indução à anestesia. No segundo, foram realizados dois testes com o objetivo de verificar a margem de segurança do anestésico. No terceiro, avaliou-se o efeito estressante do anestésico. A concentração $250 \mathrm{mg} \mathrm{L}^{-1}$ de mentol foi a mais adequada para indução de anestesia cirúrgica. Para anestesia voltada para biometria e breve inspeção visual do animal, a concentração mais adequada foi $150-200 \mathrm{mg} \mathrm{L}^{-1}$. O dobro da concentração ideal $\left(500 \mathrm{mg} \mathrm{L}^{-1}\right)$ não acarretou a morte nos indivíduos, indicando boa margem de segurança para o uso desse anestésico. $\mathrm{O}$ mentol, mesmo em sua concentração adequada, induziu ao aumento na glicose sanguínea, caracterizando estresse no peixe. Conclui-se que o mentol não é um anestésico eficaz para juvenis de tilápia durante procedimentos de rotina na piscicultura.
\end{abstract}

Palavras-chave: peixe, Oreochromis niloticus, estresse, sedação

\begin{abstract}
This work tested the effectiveness of menthol as anesthetic for Nile tilápia (Oreochromis niloticus) by the evaluation of behavioral events, the safety margin of the anesthetic, and stress response. On first experiment, six different anesthetic concentrations were tested to evaluate the induction time to anesthesia. On second experiment, two tests were carried out to verify the safety margin of the anesthetic. On third experiment, stress responses were evaluated. The safe concentration to induce a surgical anesthesia was $250 \mathrm{mg} \mathrm{L}^{-1}$. To a brief handling and visual inspection, the appropriated concentration was $150-200 \mathrm{mg} \mathrm{L}^{-1}$. There was no mortality after exposure to $500 \mathrm{mg} \mathrm{L}^{-1}$, indicating a good safety margin for this anesthetic. Menthol even in its adequate dosage induced a rise in glucose concentrations indicating a stress response of the fish. Thus menthol is an ineffective anesthetic to juveniles tilapia during routine procedures in fish culture.
\end{abstract}

Keywords: fish, Oreochromis niloticus, stress, sedation

\section{INTRODUÇ̃̃O}

A tilápia-do-nilo (Oreochromis niloticus) é o peixe mais criado no Brasil (Crescêncio, 2005). As principais razões são: a alta taxa de crescimento, a rusticidade, o hábito alimentar onívoro, a fácil aceitação de ração e a grande resistência às doenças e à baixa concentração de oxigênio na água. Além disso, a tilápia tem boa aceitação no mercado consumidor por possuir carne saborosa, de cor branca e com baixo teor de gordura (Boscolo et al., 2001).

Recebido em 24 de agosto de 2008

Aceito em 12 de fevereiro de 2009

*Autor para correspondência (corresponding author)

E-mail: levy.gomes@uvv.br 
A anestesia é um procedimento muitas vezes indispensável durante o manejo de tilápias e é realizada por diversas razões como em operações de manuseio, no transporte, em inspeções visuais e em biometria (Inoue, 2005). A anestesia de tilápia durante o manuseio é importante, uma vez que os raios duros que formam a nadadeira dorsal desta espécie facilmente ocasionam ferimentos aos operadores e aos outros peixes, caso não estejam anestesiados. Outro importante motivo para se desenvolver protocolos de anestesia para esta e outras espécies de peixe é que os comitês de ética em experimentação com animais estão solicitando procedimentos que diminuam o estresse ou proporcionem sofrimento mínimo aos animais. Ainda, o mercado de carnes para exportação pode no futuro solicitar práticas de manejo sem estresse ou com estresse mínimo.

Os produtos químicos mais comuns usados para anestesia de peixes são a tricaína metanosulfato (MS-222), a quinaldina e o 2-fenoxietanol (Ross e Ross, 1999; Mylonas et al., 2005), porém estes anestésicos são de difícil obtenção e são de alto custo (Inoue, 2005). O uso de produtos naturais, como óleos essenciais, derivados de plantas, tem se mostrado uma alternativa viável face à dificuldade de obtenção dos químicos utilizados para anestesia (Façanha e Gomes, 2005; Cunha, 2007). Os principais compostos utilizados são o eugenol, extraído das folhas e dos brotos da árvore Eugenia caryophyllata, e o mentol, extraído de plantas do gênero Mentha. Estes produtos, facilmente encontrados no mercado nacional, são de baixo custo, e sua eficiência é comprovada para o tambaqui (Colossoma macropomum), a principal espécie nativa criada no Brasil (Façanha e Gomes, 2005; Roubach et al., 2005).

O mentol é um óleo essencial com propriedades anestésicas, antiespasmódicas, anti-inflamatórias, antiúlceras e antivirais, sendo de grande importância econômica na indústria farmacêutica (Lorenzo et al., 2002) e também na alimentícia e de cosméticos. O mentol é amplamente utilizado na anestesia de invertebrados marinhos (Ruppert e Barnes, 1996), mas não é um anestésico regulamentado para uso em peixes, e nem se conhece seu período de carência no tecido dos animais. Porém, seu uso é regulamentado para humanos, muitas vezes em concentrações mais altas que as utilizadas para peixes. Essas características qualificam o mentol como um anestésico a ser testado quanto à eficiência e segurança, a fim de subsidiar as autoridades responsáveis pela regulamentação de anestésicos para peixes no Brasil (Façanha e Gomes, 2005).

Apesar de a tilápia ser o principal peixe criado no Brasil e um dos mais criados do mundo, a literatura referente ao uso de anestésicos para esta espécie é bastante limitada. Até o momento, as principais referência são para a benzocaína (Ross e Ross, 1999; Gontijo et al., 2003) e para o eugenol (Vidal et al., 2008). Assim, o objetivo deste trabalho foi testar a eficiência do mentol como anestésico para a tilápia-do-nilo, por meio da avaliação de eventos comportamentais, da verificação da margem de segurança do anestésico e das respostas de estresse.

\section{MATERIAL E MÉTODOS}

Juvenis de tilápia obtidos na piscicultura da Associação dos Aquicultores do Espírito Santo (AQUES), Cariacica, ES, foram transportadas para o Laboratório de Ecotoxicologia Aquática do Centro Universitário Vila Velha (UVV), Vila Velha, ES. Aproximadamente 150 peixes $(13,68 \pm 4,44 \mathrm{~g}$ e $9,56 \pm 1,07 \mathrm{~cm})$ foram colocados para aclimatação em um tanque de $450 \mathrm{~L}$, com sistema de aeração constante, por uma semana. Nesse período, os peixes foram alimentados diariamente com ração comercial para peixes contendo $36 \%$ de proteína bruta.

Os testes foram realizados em aquários estáticos de $13 \mathrm{~L}$, mas que continham $6 \mathrm{~L}$ de água (oxigênio dissolvido; 7,84 $\pm 0,09 \mathrm{mg} \quad \mathrm{L}^{-1}$; temperatura, $\quad 27 \pm 0,07^{\circ} \mathrm{C} ; \quad \mathrm{pH} \quad 7,13 \pm 0,03$ unidades), e a recuperação foi realizada em aquários de $30 \mathrm{~L}$, mas que continham $25 \mathrm{~L}$ de água, com aeração constante. Os cristais de mentol foram dissolvidos em uma concentração de $1 \mathrm{~g} / 10 \mathrm{~mL}$ de álcool para o preparo da solução mãe $(100 \mathrm{mg}$ de mentol para cada $\mathrm{mL}$ de solução). A água do aquário foi trocada ao término de cada teste.

Foram realizados três experimentos: o primeiro teve o objetivo de determinar a concentração adequada do anestésico; o segundo avaliou a margem de segurança do anestésico em dois diferentes testes e o terceiro avaliou o efeito estressante do anestésico. 
No primeiro experimento, foram testadas diferentes concentrações de mentol. As concentrações foram estabelecidas com base em um teste piloto, sendo a menor concentração testada $\left(50 \mathrm{mg} \mathrm{L}^{-1}\right)$ a primeira que induziu a maioria dos estágios de anestesia estudados. No experimento definitivo, foram testadas as concentrações: $50 ; 100 ; 150 ; 200 ; 250$ e $300 \mathrm{mg}$ $\mathrm{L}^{-1}$. Avaliou-se o tempo de indução à anestesia em segundos. Para cada concentração, foram utilizados 10 peixes expostos ao anestésico, de forma individual, para se observar o tempo de indução em cada estágio. Após 10 minutos de exposição ao mentol, o peixe foi removido para o aquário de recuperação. Os estágios de indução da anestesia avaliados seguiram os critérios propostos por Stoskopf (1993) (Tab. 1).

Tabela 1. Estágios de indução da anestesia em peixes

\begin{tabular}{cl}
\hline $\begin{array}{c}\text { Estágio de } \\
\text { anestesia }\end{array}$ & \multicolumn{1}{c}{\begin{tabular}{c}
\multicolumn{1}{c}{ Característica } \\
comportamental
\end{tabular}} \\
\hline 1 & $\begin{array}{l}\text { Perda de reação a estímulos } \\
\text { Perda parcial de equilíbrio }\end{array}$ \\
3 & $\begin{array}{l}\text { Perda total de equilíbrio } \\
\text { Redução dos batimentos } \\
\text { operculares } \\
\text { Parada dos batimentos } \\
\text { operculares } \\
\text { Retorno dos movimentos e } \\
\text { natação ativa }\end{array}$ \\
\hline
\end{tabular}

Adaptado de Stoskopf (1993).

No segundo experimento, foram realizados dois testes com o objetivo de verificar a margem de segurança do anestésico. Para tal, tomou-se por base o resultado do primeiro experimento, sendo eleita a concentração de $250 \mathrm{mg} \mathrm{L}^{-1}$ como a mais adequada por ter sido a que induziu ao estágio de parada dos batimentos operculares em menor tempo e provocou indução em todos os estágios de anestesia.

No primeiro teste, foi analisado o tempo de recuperação após indução à concentração de $250 \mathrm{mg} \mathrm{L}^{-1}$ de mentol por diferentes tempos. Os peixes, previamente aclimatados, foram dispostos individualmente ao anestésico por 10 , 20 e 30 minutos $(n=10$ para cada tempo de exposição). Após a exposição, o peixe foi removido para o aquário de recuperação. No segundo teste, 10 peixes foram individualmente expostos a $500 \mathrm{mg} \mathrm{L}^{-1}$ de mentol para avaliar a mortalidade na concentração duas vezes acima da ideal. Para isso, os peixes, previamente aclimatados, foram expostos ao anestésico por um período de 10 minutos. Após este período, os peixes foram transferidos para o aquário de recuperação.

No terceiro experimento, avaliou-se o efeito estressante do anestésico. Os peixes foram dispostos individualmente em 36 aquários de 30L com aeração constante, e aclimatados por 96 horas. Nesse período, foram alimentados diariamente com ração comercial contendo $36 \%$ de proteína bruta. Metade da água dos aquários foi trocada uma vez ao dia. Os peixes foram mantidos sem alimentação por 24 horas antes do experimento. Após a aclimatação, foram distribuídos em dois tratamentos, anestesiados (250mg L $\mathrm{L}^{-1}$ de mentol) e não anestesiados (controle), e em três diferentes tempos: três minutos de anestesia ( $3 \mathrm{~min}$ ), nove minutos de anestesia (9min) e 10 minutos de anestesia mais o período de recuperação $(\mathrm{Rec})$. Em cada tempo de amostragem, seis peixes foram individualmente transferidos para banho anestésico e mais seis animais do controle foram submetidos a condições semelhantes, sem o banho. Após o período de anestesia, foi coletado sangue por punção caudal, para realizar teste de glicose e hematócrito. A análise de glicose plasmática foi realizada com kit enzimático glucox 500 - Doles. Para análise de hematócrito, o sangue foi colocado em capilares e centrifugado a 3000rpm por 10 minutos, e a leitura feita em escala de hematócrito.

Os tempos para atingir os diferentes estágios de anestesia e recuperação foram avaliados para as diferentes concentrações de mentol usando-se o teste Kruskal-Wallis, a análise de variância e o teste Tukey para comparação de médias. Para o tempo de recuperação após exposição à anestesia, usou-se a análise de variância e o teste Tukey. Os resultados de glicose e hematócrito do terceiro experimento foram comparados com o respectivo grupo-controle pelo teste t. Peixes do mesmo tratamento (expostos ou controle) tiveram a glicose e hematócrito comparados nos diferentes tempos de anestesia por análise de variância e teste Tukey (Zar, 1999). As análises estatísticas foram realizadas com o programa Sigma Stat 3.0. 


\section{RESULTADOS E DISCUSSÃO}

A indução aos estágios iniciais de anestesia (perda de reações a estímulos e perda parcial de equilíbrio) foi significativamente mais rápida $\mathrm{em}$ concentrações acima de $250 \mathrm{mg} \mathrm{L}^{-1}$ (Fig. 1A e $1 \mathrm{~B})$, porém os estágios seguintes, como perda total de equilíbrio e redução dos batimentos operculares, ocorreram significativamente mais rápido em concentrações superiores a $150 \mathrm{mg} \mathrm{L}^{-1}$ (Fig. 1C e 1D). Resultados semelhantes foram obtidos por Façanha e Gomes (2005) durante a anestesia de tambaqui com mentol, em que o tempo necessário para atingir a perda total de equilíbrio e a redução dos batimentos operculares são menores a partir de concentrações acima de $150 \mathrm{mg} \mathrm{L}^{-1}$. Este resultado, quando comparado com os obtidos por Gomes et al. (2001) e Roubach et al. (2005), que utilizaram, respectivamente, eugenol e benzocaína durante a anestesia de juvenis de tambaqui, mostra que o tempo de indução à perda total de equilíbrio, estágio desejado para biometrias e breve manejo, é mais alto com o mentol.

Apenas as concentrações de 250 e $300 \mathrm{mg} \mathrm{L}^{-1}$ foram eficientes em induzir os peixes à parada dos batimentos operculares, e não houve diferença significativa entre os peixes expostos a estas duas concentrações. A mediana foi de $540 \mathrm{~s}$ e 520 s para as concentrações 250 e $300 \mathrm{mg} \mathrm{L}^{-1}$, respectivamente (Fig. 1E). Tilápias expostas a concentrações de mentol acima de $250 \mathrm{mg} \mathrm{L}^{-1}$ passam sequencialmente por todos os estágios de anestesia descritos por Stoskopf (1993). Este resultado é diferente do obtido por Façanha e Gomes (2005), em que juvenis de tambaqui expostos a baixas concentrações $\left(50 \mathrm{mg} \mathrm{L}^{-1}\right)$ de mentol passam por todos os estágios de anestesia. Juvenis de bagre do canal (Ictalurus punctatus) e truta arco-íris (Oncorhynchus mykiss) anestesiados com óleo de cravo (eugenol) também são induzidos a todos os estágios de anestesia com concentrações baixas (Keene et al., 1998; Waterstrat, 1999).

A recuperação dos peixes expostos a $50 \mathrm{mg} \mathrm{L}^{-1}$ foi significativamente menor que a obtida com os peixes expostos a 100, 150, 200, 250 e $300 \mathrm{mg} \mathrm{L}^{-1}$. Os expostos a 50 e $250 \mathrm{mg} \mathrm{L}^{-1}$ apresentaram recuperação em $317,5 \mathrm{~s}$ e $805 \mathrm{~s}$, respectivamente (Fig. 1F).

A concentração de $250 \mathrm{mg} \mathrm{L}^{-1}$ de mentol é a mais adequada para indução de anestesia cirúrgica em tilápia, pois é a que induz o estágio de parada dos batimentos operculares em menor tempo. Para anestesia voltada para biometria e breve manejo, o estágio de anestesia desejado é a perda total de equilíbrio. Dessa forma, a concentração recomendada é $150-200 \mathrm{mg} \mathrm{L}^{-1}$, pois os peixes são induzidos a este estágio de anestesia, em tempos semelhantes à concentração de $250 \mathrm{mg} \mathrm{L}^{-1}$. Façanha e Gomes (2005) encontraram resultado diferente para o tambaqui, isto é, as concentrações adequadas para anestesia voltada para cirurgia e biometria e breve manejo são, respectivamente, $100 \mathrm{e} 150 \mathrm{mg} \mathrm{L}^{-1}$ de mentol, ou seja, cerca de $50 \%$ mais baixas que as obtidas para tilápia.

Após a exposição por 10,20 e 30 minutos, a $250 \mathrm{mg} \mathrm{L}^{-1}$ de mentol, a tilápia nilótica apresenta diferença significativa entre os tempos de recuperação (Fig. 2). O tempo para recuperação dos peixes expostos por 10 minutos é significativamente menor que o dos peixes expostos por 20 e 30 minutos. A média de tempo para a recuperação após a exposição por 10, $20 \mathrm{e}$ 30 minutos foi de $548,4 \pm 72,85$ segundos, $753,8 \pm 195,88$ segundos e $803,4 \pm 161,33$ segundos, respectivamente. Houve mortalidade de 20 e $10 \%$ dos peixes expostos por 20 e 30 minutos, respectivamente. Ross e Ross (1999) e Roubach e Gomes (2001) acreditam que 10 minutos deve ser o tempo máximo de anestesia. Os resultados obtidos neste estudo confirmam isso, pois a margem de segurança do mentol para uma exposição prolongada ( 20 e 30 minutos) é baixa. Apesar de a tilápia não resistir à exposição de $250 \mathrm{mg} \mathrm{L}^{-1}$ por tempos acima de 10 minutos, não há mortalidade quando anestesiada por este tempo com o dobro da concentração ideal. Este resultado mostra que a margem de segurança para a anestesia de até 10 minutos é bastante alta. 
Eficácia do mentol como anestésico...
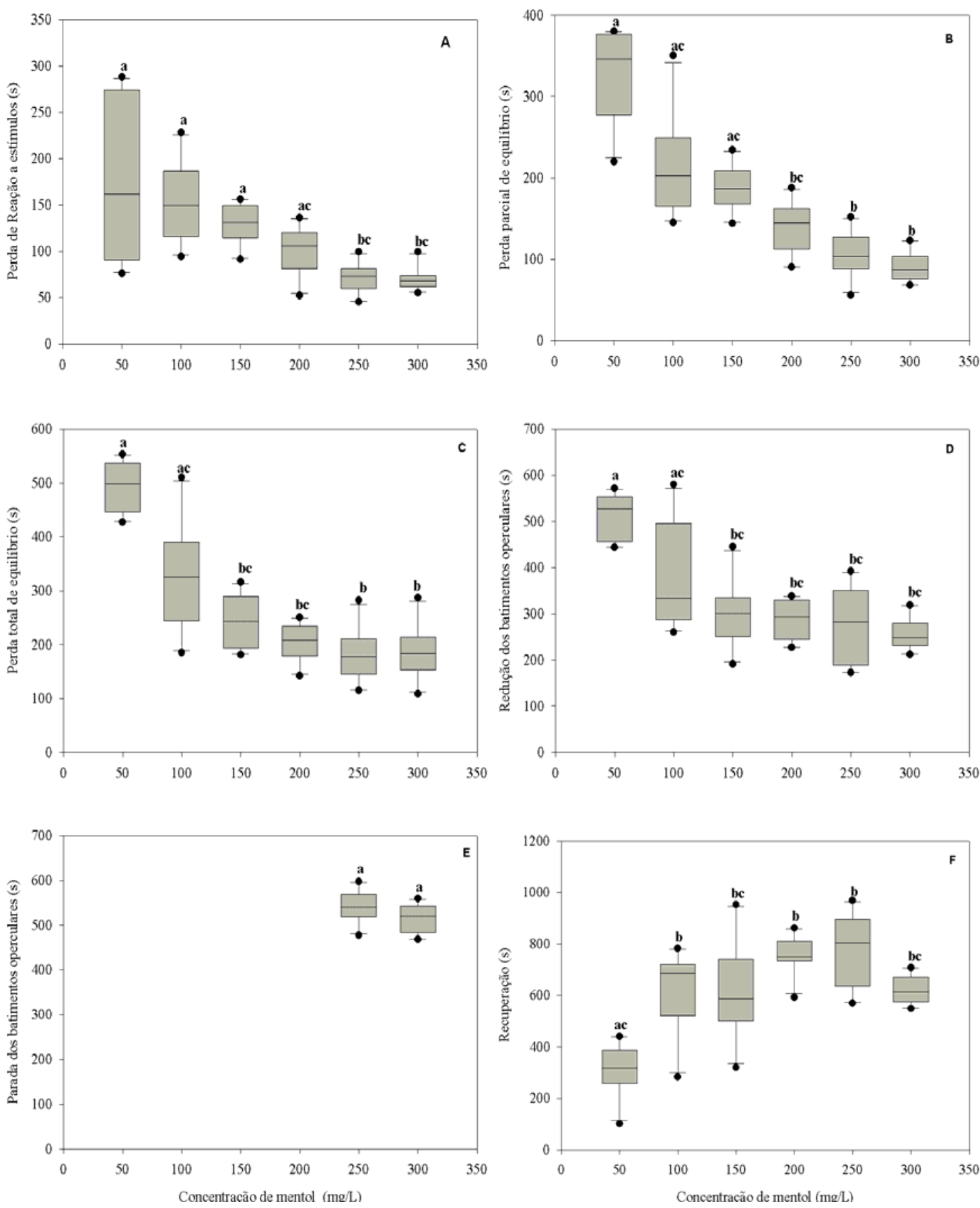

Figura 1. Box plot dos tempos, em segundos (s), de indução aos estágios de anestesia de tilápias expostas a diferentes concentrações de mentol. Mediana do tempo necessário para atingir os estágios de anestesia marcada por diferentes letras são significativamente diferentes (Kruskal-Wallis e teste Tukey; $\mathrm{P}<0,05$ ). 


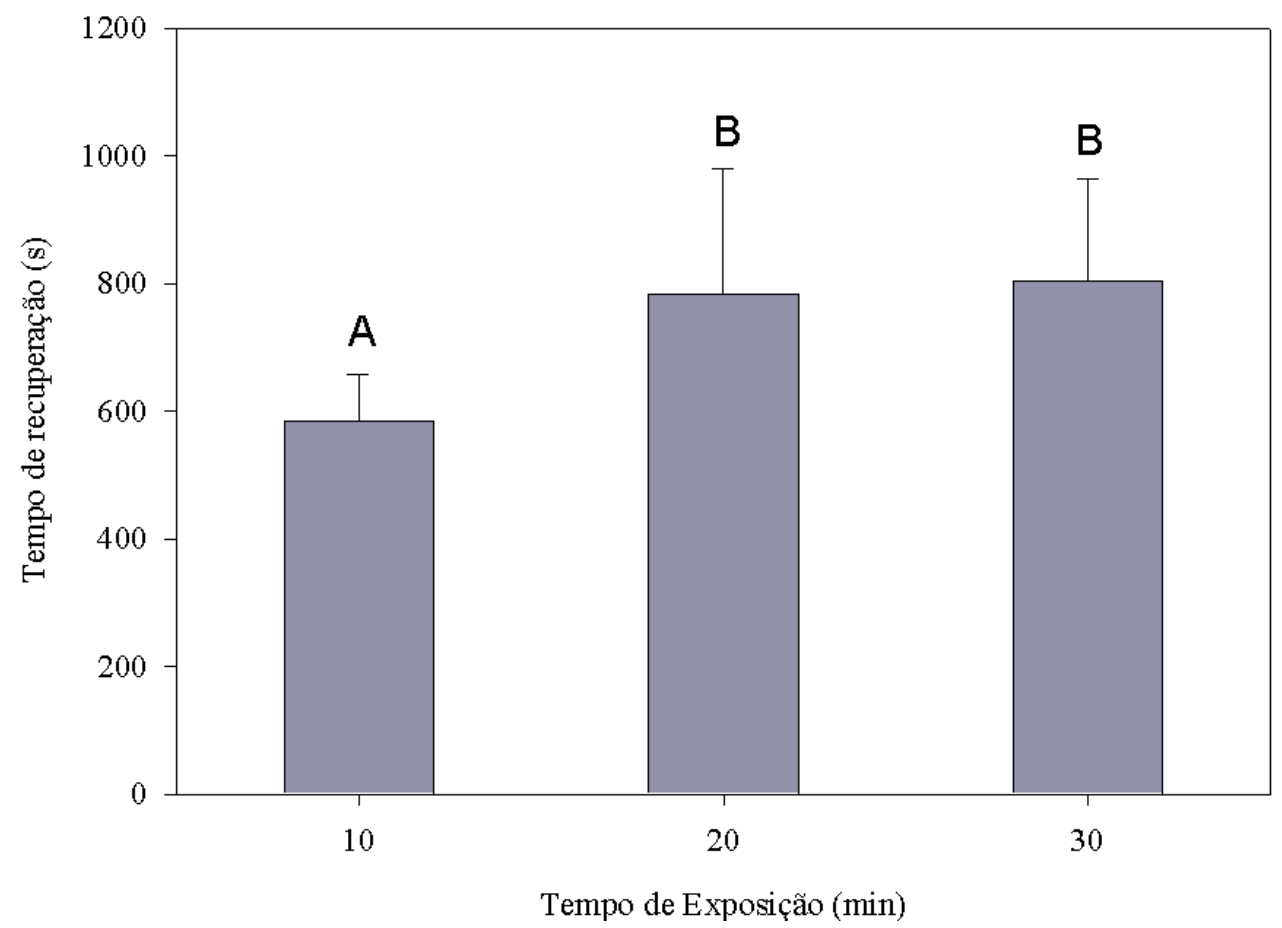

Figura 2. Tempo de recuperação de tilápia nilótica após a exposição por diferentes tempos a $250 \mathrm{mg} \mathrm{L}^{-1} \mathrm{de}$ mentol. Letras diferentes nas colunas significam diferença significativa por análise de variância e teste Tukey $(\mathrm{P}<0.05)$.

Os anestésicos atuam nos peixes deprimindo as funções neurossensoriais. A maior parte dos anestésicos afeta o sistema nervoso central e atua primeiro sobre o córtex cerebral. $\mathrm{O}$ aumento da concentração ou do tempo de exposição difunde os seus efeitos através do tronco cerebral para o centro respiratório medular e da medula espinhal (Summerfelt e Smith, 1990). Dessa forma, a indução à anestesia ocorre em efeito cascata, como descrito na Tab. 1. De acordo com Summerfelt e Smith (1990), a exposição a um anestésico pode alterar as respostas de estresse.

A glicose plasmática é bom indicador de estresse, pois as alterações são facilmente detectáveis e sua avaliação pode ser realizada com medidores de glicose de simples utilização (Gomes et al., 2005). A glicose dos peixes expostos ao anestésico por diferentes tempos (3min, 9min e recuperação) é significativamente maior do que a do seu respectivo controle (Fig. 3). A glicose plasmática dos peixes expostos por 3 min foi significativamente menor do que a dos peixes expostos por 9 min e na recuperação, e não houve diferença significativa na glicose dos peixes expostos por 9 min e na recuperação. Os resultados indicaram que a tilápia exposta ao mentol apresenta aumento significativo na concentração de glicose plasmática em todos os tempos de amostragem. Este resultado mostra que a exposição ao mentol é estressante aos peixes. Isto foi observado por Inoue (2005) para o eugenol e o 2-fenoxietanol, durante a anestesia de matrinxã (Brycon amazonicus). A glicose plasmática do grupo-controle manteve-se constante durante os diferentes tempos de amostragens, e os valores obtidos estão próximos aos relatados por Martins et al. (2004) como basais para esta espécie.

O hematócrito não apresentou diferença significativa entre os peixes anestesiados e controle em nenhum tempo de amostragem (Fig. 3), assim como não houve diferença entre os peixes anestesiados nos diferentes tempos de amostragem. $\mathrm{O}$ mesmo resultado foi obtido com os peixes-controle. De acordo com Morales et al. (2005), mudanças nas respostas hematológicas de estresse, como o hematócrito, normalmente não são observadas em exposições agudas, sendo mais responsivo em exposições crônicas. 

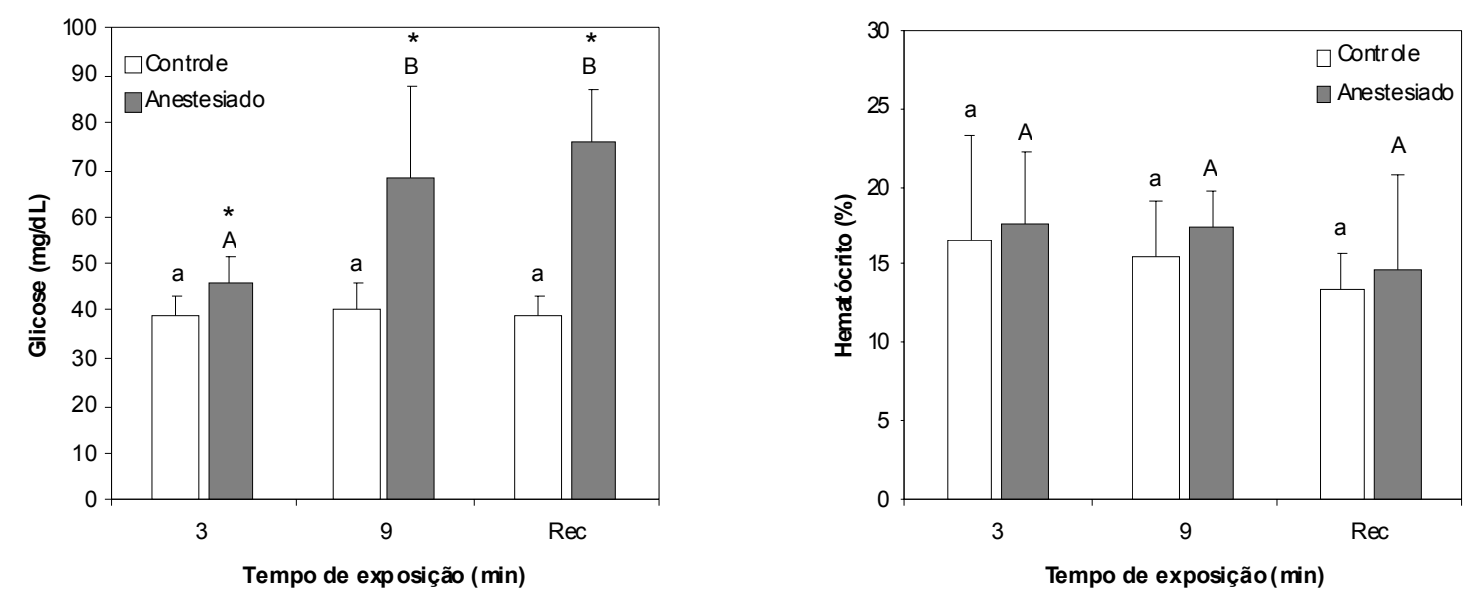

Figura 3. Concentrações de glicose plasmática e hematócrito em tilápia nilótica após a exposição por diferentes tempos a $250 \mathrm{mg} \mathrm{L}^{-1}$ de mentol. Rec $=10$ minutos de anestesia +recuperação. * indica diferença significativa do respectivo controle em cada tempo de exposição. Letras minúsculas indicam diferença significativa do tratamento-controle nos diferentes tempos de exposição por análise de variância e teste Tukey $(\mathrm{P}<0.05)$. Letras maiúsculas indicam diferença significativa do tratamento anestesiado nos diferentes tempos de exposição por análise de variância e teste Tukey $(\mathrm{P}<0.05)$.

\section{CONCLUSÕES}

O mentol, em sua concentração adequada para indução à anestesia, causa um aumento na glicose sanguínea, caracterizando estresse no peixe, não sendo, portanto, um anestésico preferencial para uso com a tilápia-do-nilo.

\section{AGRADECIMENTOS}

Trabalho financiado por projeto interno do Centro Universitário de Vila Velha (\#16/2008). L.C. Gomes e bolsistas de produtividade científica do CNPq.

\section{REFERÊNCIAS BIBLIOGRÁFICAS}

BOSCOLO, W.R.; HAYASHI, C.; SOARES, C.M. et al. Desempenho e características de carcaça de machos revertidos de tilápias do nilo (Oreochromis niloticus), linhagens tailandesa e comum, nas fases inicial e de crescimento. Rev. Bras. Zootec., v.30, p.1391-1396, 2001.

CRESCÊNCIO, R. Ictiofauna brasileira e seu potencial para criação. In: BALDISSEROTTO, B.; GOMES, L.C. (Eds). Espécies nativas para piscicultura no Brasil. Santa Maria: UFSM, 2005. p.23-33.
CUNHA, M.A. Tempo de indução e recuperação anestésica em jundiás (Rhamdia quelen) expostos a substâncias isoladas de plantas. 2007. 63f. Dissertação (Mestrado) - Universidade Federal de Santa Maria, Santa Maria, RS.

FAÇANHA, M.F.; GOMES, L.C. A eficácia do mentol como anestésico para tambaqui (Colossoma macropomum, Characiformes: Characidae). Acta Amazônica, v.35, p.71-75, 2005.

GOMES, L.C.; CHIPPARI-GOMES, A.R.; LOPES, N.P. et al. Efficacy of benzocaine as an anesthetic in juvenile tambaqui, Colossoma macropomum. J. World Aquacult. Soc., v.32, p.426-431, 2001.

GOMES, L.C.; CHAGAS, E.C.; CRESCÊNCIO, R. et al. Validation of a simple portable instrument for measurement of blood glucose in four amazon fishes. J. Aquacult. Trop., v.20, p.101-109, 2005.

GONTIJO, A.M.M.C.; BARRETO, R.E.; SPEIT, G. et al. Anesthesia of fish with benzocaine does not interfere with comet assay results. Mutat. Res., v.534, p.175-162, 2003.

INOUE, L.A.K.A. Respostas do matrinxã (Brycon cephalus) a anestésicos e estressores. 2005. 167f. Tese (Doutorado) - Universidade Federal de São Carlos, São Carlos, SP. 
KEENE，J.L.; NOAKES, D.L.G.; MOCCIA, R.D. et al. The efficacy of clove oil as an anaesthetic for rainbow trout, Oncorhynchus mykiss (Walbaum). Aquacult. Res., v.29, p.89$101,1998$.

LORENZO, D.; PAZ, D.; DELLACASSA, E. et al. Essential oils of Mentha pulegium and Mentha rotundifolia from Uruguay. Braz. Arch. Biol. Technol., v.45, p.519-524, 2002.

MARTINS, M.L.; PILARSKY, F.; ONAKA, E.M. et al. Hematologia e resposta inflamatória aguda em Oreochromis niloticus (Osteichthyes: Cichlidae) submetida aos estímulos único e consecutivo de estresse de captura. Bol. Inst. Pesca, v.30, p.71-80, 2004.

MYLONAS, C.C.; CARDINALETTI, G.; IGELAKI, I. et al. Comparative efficacy of clove oil and 2-phenoxyethanol as anesthetics in the aquaculture of European sea bass (Dicentrarchus labrax) and gilthead sea bream (Sparus aurata) at different temperatures. Aquaculture, v.246, p.467-481, 2005.

MORALES, A.E.; CARDENETE, G.; ABELLÁN, E. et al. Stress-related physiological responses to handling in common dentex (Dentex dentex Linnaeus, 1758). Aquacult. Res., v.36, p.33-40, 2005.

ROSS, L.G.; ROSS, B. Anaesthetic \& sedative techniques for aquatic animals. Oxford: Blackwell Science, 1999. 159p.
ROUBACH, R.; GOMES, L.C. O uso de anestésicos durante o manejo de peixes. Pan. Aquicult., v.11, p.37-40, 2001.

ROUBACH, R.; GOMES, L.C.; FONSECA, F.A.L. et al. Eugenol as an efficacious anaesthetic for tambaqui, Colossoma macropomum (Cuvier). Aquacult. Res., v.36, p.16, 2005.

RUPPERT, E.E.; BARNES, R.D. Zoologia dos invertebrados. 6.ed. São Paulo: Roca, 1996. $1029 \mathrm{p}$.

SUMMERFELT, R.C.; SMITH, L.S. Anesthesia, surgery and related techniques. In: SCHRECK, C.B.; MOYLE, P.B. (Eds.) Methods for fish biology. Maryland, USA: American Fisheries Society, 1990. p.213-278.

STOSKOPF, M. Anaesthesia. In: BROWN, L. (Ed). Aquaculture for Veterinarians: fish husbandry and medicine. London, UK: Pergamon Veterinary Handbook Series, 1993. p.161-168.

VIDAL, L.V.O.; ALBINATI, R.C.B.; ALBINATI, A.C.L. et al. Eugenol como anestésico para a tilapia-do-nilo. Pesq. Agropec. Bras., v.43, p.1069-1074, 2008.

WATERSTRAT, P.R. Induction and recovery from anesthesia in channel catfish Ictalurus punctatus fingerlings exposed to clove oil. $J$. World Aquacult. Soc., v.30, p.250-255, 1999.

ZAR, J.H. Biostatiscal analysis. 4.ed. Upper Saddler River: Prentice Hall, 1999. 663p. 